PROCEEDINGS OF THE

AMERICAN MATHEMATICAL SOCIETY

Volume 136, Number 7, July 2008, Pages 2271-2278

S 0002-9939(08)09466-5

Article electronically published on March 6, 2008

\title{
SURFACES VIOLATING BOGOMOLOV-MIYAOKA-YAU IN POSITIVE CHARACTERISTIC
}

\author{
ROBERT W. EASTON \\ (Communicated by Ted Chinburg)
}

\begin{abstract}
The Bogomolov-Miyaoka-Yau inequality asserts that the Chern numbers of a surface $X$ of general type in characteristic 0 satisfy the inequality $c_{1}^{2} \leq 3 c_{2}$, a consequence of which is $\frac{K_{X}^{2}}{\chi\left(\mathcal{O}_{X}\right)} \leq 9$. This inequality fails in characteristic $p$, and here we produce infinite families of counterexamples for large $p$. Our method parallels a construction of Hirzebruch, and relies on a construction of abelian covers due to Catanese and Pardini.
\end{abstract}

\section{INTRODUCTION}

In 1976, Bogomolov [B] proved that if $X$ is a complex surface of general type, then the Chern numbers of $X$ satisfy the inequality $c_{1}^{2} \leq 4 c_{2}$, where $c_{i}=c_{i}\left(\mathcal{T}_{X}\right)$. This inequality was improved independently by Miyaoka [M2] and Yau $[\mathrm{Y}]$ to $c_{1}^{2} \leq 3 c_{2}$, which is sharp by examples of Hirzebruch [H1]-[H3]. A consequence of this latter inequality is that if $X$ is a surface of general type in characteristic 0 , then $\frac{K_{X}^{2}}{\chi\left(\mathcal{O}_{X}\right)} \leq 9$. This inequality does not hold in positive characteristic, as can already be seen in families of surfaces constructed by Lang $[\mathrm{L}$. In this paper we produce infinite families of counterexamples in large characteristics.

The surfaces we generate parallel a construction of Hirzebruch. In [H2], Hirzebruch produced smooth complex surfaces of general type with $c_{1}^{2}=3 c_{2}$ by constructing abelian covers of $\mathbb{P}^{2}$ branched over specific configurations of lines. Here we apply a similar procedure in positive characteristic, producing surfaces branched over pathological configurations of lines unique to positive characteristic.

More explicitly, following the notation of Vakil [V, §4], fix a prime $q$ and positive integer $n$, and let $G=(\mathbb{Z} / q \mathbb{Z})^{n}$. Let $\langle\cdot, \cdot\rangle: G \times G^{\vee} \rightarrow \mathbb{Z} / q \mathbb{Z}$ be the natural pairing, which we extend in the obvious way to a map $\langle\cdot, \cdot\rangle: G \times G^{\vee} \rightarrow \mathbb{Z}$. (Note that this map is no longer bilinear, but it shall not be needed here.) Suppose $k$ is a field of characteristic $p \neq q$, and let $S$ be the blowup of $\mathbb{P}_{k}^{2}$ at the $\left(p^{2}+p+1\right) \mathbb{F}_{p}$-valued points. Suppose we have two maps:

$$
\begin{aligned}
& D: G \rightarrow \operatorname{Div}(S), \\
& L: G^{\vee} \rightarrow \operatorname{Pic}(S) .
\end{aligned}
$$

Received by the editors December 6, 2005.

2000 Mathematics Subject Classification. Primary 14J29.

Key words and phrases. Bogomolov inequality, abelian cover, positive characteristic, algebraic surface, general type.

(C)2008 American Mathematical Society Reverts to public domain 28 years from publication 
We say $(D, L)$ satisfy the cover condition if $D_{0}=0$ and if, for all $\chi \in G^{\vee}$,

$$
q L_{\chi}=\sum_{\sigma \in G}\langle\chi, \sigma\rangle D_{\sigma},
$$

with equality taking place in $\operatorname{Pic}(S)$.

We then have the following result, due to Catanese [C] and Manetti [M1, §3] in the case $G=(\mathbb{Z} / 2 \mathbb{Z})^{n}$ and Pardini $[\mathrm{P}, \S 4]$ in general:

Proposition A. Suppose $(D, L)$ satisfy the cover condition, and

(i) $D_{\sigma}$ are all nonsingular curves;

(ii) no three meet at a point; and

(iii) if $D_{\sigma}, D_{\sigma^{\prime}}$ meet, then they are transverse, and $\sigma, \sigma^{\prime}$ are independent in $G$.

Then:

(1) There exists a $G$-cover $\pi: \tilde{S} \rightarrow S$ with $\tilde{S}$ a nonsingular surface, and with branch locus $D=\bigcup D_{\sigma}$;

(2) $q^{n} K_{\tilde{S}}=\pi^{*}\left(q^{n} K_{S}+q^{n-1}(q-1) \sum_{\sigma} D_{\sigma}\right)$, with equality holding in $\operatorname{Pic}(\tilde{S})$;

(3) $K_{\tilde{S}}^{2}=q^{n}\left(K_{S}+\frac{q-1}{q} \sum_{\sigma} D_{\sigma}\right)^{2} ;$ and

(4) $\chi\left(\mathcal{O}_{\tilde{S}}\right)=q^{n} \chi\left(\mathcal{O}_{S}\right)+\frac{1}{2} \sum_{\chi} L_{\chi} \cdot\left(L_{\chi}+K_{S}\right)$.

Our general strategy is to build surfaces $\tilde{S}$ using divisors on $S$ which exploit pathologies in positive characteristic. One such pathology is the following: If we consider the set of lines through pairs of $\mathbb{F}_{p}$-valued points in $\mathbb{P}_{k}^{2}$, we find there are more such lines than "would be expected" in the characteristic 0 case. To exploit this pathology, we let $C$ be the union of these lines and take the strict transform $\tilde{C}$ of $C$ to be one of our divisors. We then choose the remaining divisors to be unions of appropriate numbers of pullbacks of general lines in $\mathbb{P}^{2}$. With particularly cogent choices of divisors, line bundles $L_{\chi}$ can be defined directly from the covering condition.

Our main result is the following:

Theorem. For each prime $q \geq 3$ and all sufficiently large primes $p \equiv-1(\bmod q)$, there exists a surface $\tilde{S}$ in characteristic $p$, nonsingular and of general type, with $\frac{K_{\tilde{S}}^{2}}{\chi\left(\mathcal{O}_{\tilde{S}}\right)}>9$.

\section{The COnstruction}

Let $p, q$ be primes with $q \geq 3$ and $p \equiv-1(\bmod q)$, and let $n \geq 3$ be any integer.

For each $\sigma \in G=(\mathbb{Z} / q \mathbb{Z})^{n}$, write $\sigma=\left(\sigma_{1}, \ldots, \sigma_{n}\right)$, with $\sigma_{i} \in\{0, \ldots, q-1\}$. Let $\vec{e}_{1}, \ldots, \vec{e}_{n}$ represent the standard unit 'vectors'. Also, for each $\gamma \in G$, let $\chi_{\gamma} \in G^{\vee}$ be defined by $\left\langle\chi_{\gamma}, \vec{e}_{i}\right\rangle=\gamma_{i}$. Throughout, let $k$ be a field with $\operatorname{char}(k)=p$.

Observe that there are $\left(p^{2}+p+1\right) \mathbb{F}_{p}$-valued points in $\mathbb{P}_{k}^{2}$, and the same number of such lines. Also observe that through any fixed $\mathbb{F}_{p}$-valued point there pass exactly $p+1$ of these lines, and each line contains exactly $p+1$ of the $\mathbb{F}_{p}$-valued points. Let $S$ be the blowup of $\mathbb{P}_{k}^{2}$ at the $\mathbb{F}_{p^{-v a l u e d}}$ points, and let $\tilde{C}$ be the strict transform of the union $C$ of the lines through these points. Note that $K_{S} \sim-3 H+\sum_{i=1}^{p^{2}+p+1} E_{i}$ and $[\tilde{C}] \sim\left(p^{2}+p+1\right) H-(p+1) \sum E_{i}$, where $H$ is the hyperplane class of $S$ and the $E_{i}$ are the exceptional divisors. 
We will set $D_{\vec{e}_{1}}=[\tilde{C}]$, and choose the remaining divisors $D_{\sigma}$ to be the union of the pullback of general lines in $\mathbb{P}^{2}$. To ensure that the covering condition is 'solvable', some care is needed in choosing the number of sections for each divisor. To that end, let $F \subset G$ be any subset such that $\left\{\overrightarrow{0}, \vec{e}_{1}+\vec{e}_{2}, \vec{e}_{1}, \ldots, \vec{e}_{n}\right\} \subset F$, $F \cap k F=\{\overrightarrow{0}\}$ for $k=2, \ldots, q-1$, and $G=\bigcup_{k=1}^{q-1} k F$. More concretely, $F$ should contain the required elements and exactly one representative from each distinct subset of the form $\{k \sigma: k=1, \ldots, q-1\}$.

Lemma 1. There exists a collection of integers $m_{\sigma} \in\{0, \ldots, q-1\}$ such that

(i) $m_{\vec{e}_{1}}=1$;

(ii) $m_{\sigma}=0$, for all $\sigma \notin F$; and

(iii) $\sum_{\sigma \in G} m_{\sigma} \sigma=\overrightarrow{0}$.

Proof. Define the set $\tilde{F}=F \backslash\left\{\overrightarrow{0}, \vec{e}_{1}+\vec{e}_{2}, \vec{e}_{1}, \ldots, \vec{e}_{n}\right\}$. Let $m_{\sigma}=0$ for all $\sigma \notin F$, $m_{\sigma}=1$ for all $\sigma \in \tilde{F}, m_{\overrightarrow{0}}=0$, and $m_{\vec{e}_{1}}=1$. Consider the element

$$
\left(A_{1}, \ldots, A_{n}\right):=\sum_{\sigma \in \tilde{F}} \sigma \in G
$$

Working modulo $q$, choose $m_{\vec{e}_{1}+\vec{e}_{2}} \equiv-A_{1}-1, m_{\vec{e}_{2}} \equiv-A_{2}+A_{1}+1$, and $m_{\vec{e}_{i}} \equiv-A_{i}$ for each $i=3, \ldots, n$. The result follows.

Let $\mathfrak{S}$ be any collection of such integers. We're then in a position to choose our divisors and line bundles. For a fixed choice of $r_{\sigma} \in \mathbb{Z}_{\geq 0}$, let

- $D_{\overrightarrow{0}}=0$,

- $D_{\overrightarrow{e_{1}}}=[\tilde{C}]$,

- $D_{\sigma}= \begin{cases}\text { union of } q r_{\sigma}+m_{\sigma} \text { general sections of } S, & \text { if } \sigma \in F \backslash\left\{\overrightarrow{0}, \overrightarrow{e_{1}}\right\}, \\ 0, & \text { otherwise. }\end{cases}$

Note that

$$
\begin{aligned}
& \text { - } D_{\overrightarrow{e_{1}}} \sim\left(p^{2}+p+1\right) H-(p+1) \sum_{i=1}^{p^{2}+p+1} E_{i}, \\
& \text { - } D_{\sigma} \sim \begin{cases}\left(q r_{\sigma}+m_{\sigma}\right) H, & \text { if } \sigma \in F \backslash\left\{\overrightarrow{0}, \overrightarrow{e_{1}}\right\}, \\
0, & \text { otherwise. }\end{cases}
\end{aligned}
$$

Our choice of the sets $F, \mathfrak{S}$ ensures that conditions (i)-(iii) of Proposition A are satisfied. Moreover, from the lemma (and the fact that $p \equiv-1(\bmod q))$ it is straightforward to verify that $q \mid \sum_{\sigma \in G}\left\langle\chi_{\gamma}, \sigma\right\rangle D_{\sigma}$, for every $\gamma \in G$. We may therefore define

$$
L_{\chi_{\gamma}}:=\frac{1}{q} \sum_{\sigma \in G}\left\langle\chi_{\gamma}, \sigma\right\rangle D_{\sigma}
$$

for each $\gamma \in G$, which clearly satisfy the covering condition. For later reference, these choices of $D_{\sigma}$ and $L_{\chi}$ give

$$
\sum_{\sigma \in G} D_{\sigma} \sim\left(p^{2}+p+1+\sum_{\sigma \in F \backslash\left\{\overrightarrow{0}, \overrightarrow{e_{1}}\right\}}\left(q r_{\sigma}+m_{\sigma}\right)\right) H-(p+1) \sum_{i=1}^{p^{2}+p+1} E_{i}
$$


and

$$
\begin{aligned}
& \sum_{\gamma \in G} L_{\chi_{\gamma}} \cdot\left(L_{\chi_{\gamma}}+K_{S}\right) \\
&=\frac{1}{q^{2}} \sum_{\gamma \in G}\left[\left(\gamma_{1}\left(p^{2}+p+1\right)+f(\gamma, \sigma)\right) \cdot\left(-3 q+\gamma_{1}\left(p^{2}+p+1\right)+f(\gamma, \sigma)\right)\right. \\
&\left.\quad+\gamma_{1}(p+1)\left(q-\gamma_{1}(p+1)\right)\left(p^{2}+p+1\right)\right],
\end{aligned}
$$

where $f(\gamma, \sigma)=\sum_{\sigma \in F \backslash\left\{\overrightarrow{0}, \overrightarrow{e_{1}}\right\}} \overline{\gamma \cdot \sigma}\left(q r_{\sigma}+m_{\sigma}\right)$, and where $\overline{\gamma \cdot \sigma}$ is the reduced residue of $\gamma \cdot \sigma$ modulo $q$.

Before continuing, we should check that the surface $\tilde{S}$ constructed from these $(D, L)$ is of general type.

Lemma 2. Let $\tilde{S}$ be the abelian cover of $S$ with building data $(D, L)$ given above. Then $\tilde{S}$ is of general type.

Proof. Recall that $\tilde{S}$ is of general type if and only if the divisor $K_{\tilde{S}}$ is big (and that on a smooth projective surface, a divisor $D$ is big if for some integer $n>0$ one has $n D \sim E+A$ for some effective divisor $E$ and ample divisor $A$ ). Since $q^{n} K_{\tilde{S}}=\pi^{*}\left(q^{n} K_{S}+q^{n-1}(q-1) \sum_{\sigma} D_{\sigma}\right)$ and $\pi$ is a finite morphism, it suffices to prove that the divisor $q^{n} K_{S}+q^{n-1}(q-1) \sum_{\sigma} D_{\sigma}$ is big. By our choice of divisors $D_{\sigma}$, we have

$$
\begin{aligned}
q^{n} K_{S} & +q^{n-1}(q-1) \sum_{\sigma} D_{\sigma} \sim q^{n}\left(-3 H+\sum_{i} E_{i}\right) \\
& +q^{n-1}(q-1)\left(\left(p^{2}+p+1+\sum_{\sigma \in F \backslash\left\{\overrightarrow{0}, \overrightarrow{e_{1}}\right\}}\left(q r_{\sigma}+m_{\sigma}\right)\right) H-(p+1) \sum_{i} E_{i}\right) \\
\sim & q^{n-1}\left(-3 q+(q-1)\left(p^{2}+p+1+\sum_{\sigma \in F \backslash\left\{\overrightarrow{0}, \overrightarrow{e_{1}}\right\}}\left(q r_{\sigma}+m_{\sigma}\right)\right)\right) H \\
& +q^{n-1}(q-(q-1)(p+1)) \sum_{i} E_{i} q_{i}^{n-1}(q-1)\left(\left(p^{2}+p+1\right) H-(p+1) \sum_{i} E_{i}\right) \\
& +q^{n-1}\left((q-1) \sum_{\sigma \in F \backslash\left\{\overrightarrow{0}, \overrightarrow{e_{1}}\right\}}\left(q r_{\sigma}+m_{\sigma}\right)-3 q\right) H+q^{n} \sum_{i} E_{i} .
\end{aligned}
$$

Now, since $r_{\sigma}, m_{\sigma} \geq 0$ for all $\sigma$ and $m_{\sigma}=1$ for all $\sigma \in \tilde{F}$, we have

$$
\sum_{\sigma \in F \backslash\left\{\overrightarrow{0}, \vec{e}_{1}\right\}}\left(q r_{\sigma}+m_{\sigma}\right) \geq|\tilde{F}|=\frac{q^{n}-1}{q-1}-n,
$$


and so

$(q-1) \sum_{\sigma \in F \backslash\left\{\overrightarrow{0}, \overrightarrow{e_{1}}\right\}}\left(q r_{\sigma}+m_{\sigma}\right)-3 q \geq q^{n}-1-(q-1) n-3 q=q^{n}-(n+3) q+n-1>0$

(for $q, n \geq 3$ ). We can therefore write

$$
q^{n} K_{S}+q^{n-1}(q-1) \sum_{\sigma} D_{\sigma} \sim q^{n-1}(q-1)[\tilde{C}]+m H+q^{n} \sum E_{i}
$$

for some $m>0$. Since $q^{n-1}(q-1)[\tilde{C}]+q^{n} \sum E_{i}$ is effective and $H$ is ample, the result follows.

\section{REsults}

Using equations (11) and (2) above, it is straightforward to calculate $\frac{K^{2}}{\chi}$ for given values of $q, n$ and $p$. For simplicity, for the remainder of this paper we'll assume $r_{\sigma}=1$ for all $\sigma \in G$. With this convention and the above notation, let us define

$$
R(q, n, p ; F, \mathfrak{S}):=\frac{K_{\tilde{S}}^{2}}{\chi\left(\mathcal{O}_{\tilde{S}}\right)} .
$$

This ratio will clearly depend on the particular choice of the sets $F$ and $\mathfrak{S}$. As we will see, however, our main result will be independent of these choices.

Before proceeding to the main result, let us first give a few explicit calculations, as examples of the types of ratios one obtains. In the following examples, the set $F$ was canonically chosen (via a construction based on the natural ordering of elements in $\left.(\mathbb{Z} / q \mathbb{Z})^{n}\right)$, and the set $\mathfrak{S}$ was then consistently chosen as in the proof of Lemma 1. (It is not worth going into the specifics of the construction, since it is used only in the following examples and will not be needed for any of the later results.)

For $n=3$ we found:

$$
\begin{aligned}
& R(3,3, p)=\frac{3\left(7568+351 p+351 p^{2}+8 p^{3}\right)}{3023+134 p+134 p^{2}+2 p^{3}}, \\
& R(5,3, p)=\frac{497024+5647 p+5647 p^{2}+24 p^{3}}{63197+708 p+708 p^{2}+2 p^{3}} \\
& R(7,3, p)=\frac{7112888+32015 p+32015 p^{2}+48 p^{3}}{896249+4006 p+4006 p^{2}+4 p^{3}} .
\end{aligned}
$$

As another example, for $n=4$ we found:

$$
\begin{aligned}
& R(3,4, p)=\frac{3\left(91808+1215 p+1215 p^{2}+8 p^{3}\right)}{35045+458 p+458 p^{2}+2 p^{3}}, \\
& R(5,4, p)=\frac{13727024+29647 p+29647 p^{2}+24 p^{3}}{1721447+3708 p+3708 p^{2}+2 p^{3}}, \\
& R(7,4, p)=\frac{365995160+229583 p+229583 p^{2}+48 p^{3}}{45800437+28702 p+28702 p^{2}+4 p^{3}} .
\end{aligned}
$$


More relevant than the ratios themselves, however, will be their behavior as we let $p$ tend to infinity. So, let us define

$$
R(q, n):=\lim _{p \rightarrow \infty} R(q, n, p) .
$$

From our above list, we then see that

$$
R(3,3)=R(3,4)=R(5,3)=R(5,4)=R(7,3)=R(7,4)=12 .
$$

Let us now prove the following:

Proposition. For all primes $q \geq 3$, and integers $n \geq 3$,

$$
R(q, n)=12,
$$

independent of the choices of the sets $F, \mathfrak{S}$.

The bulk of the proof is contained in the following lemma.

Lemma 3. For fixed $q$ and $n$, we have the following estimates, independent of the choices of the sets $F, \mathfrak{S}$ :

(i) $K_{S}^{2}=O\left(p^{2}\right)$;

(ii) $K_{S} \cdot \sum_{\sigma} D_{\sigma}=p^{3}+O\left(p^{2}\right)$;

(iii) $\left(\sum_{\sigma} D_{\sigma}\right)^{2}=-p^{3}+O\left(p^{2}\right)$;

(iv) $\sum_{\chi} L_{\chi} \cdot\left(L_{\chi}+K_{S}\right)=\left(\frac{1}{6} q^{n-2}\left(q^{2}-1\right)\right) p^{3}+O\left(p^{2}\right)$.

Proof. First note that the sizes of the sets $F$ and $\mathfrak{S}$ are bounded in terms of $q$ and $n$, as are also the elements of $\mathfrak{S}$. Thus, for fixed $q$ and $n$, sums over $F$ of elements in $\mathfrak{S}$ contribute $O(1)$. This observation considerably simplifies many of the following estimates.

(i) Since $K_{S}=-3 H+\sum_{i=1}^{p^{2}+p+1} E_{i}$, we immediately have $K_{S}^{2}=9-\left(p^{2}+p+1\right)=$ $O\left(p^{2}\right)$. For the remaining estimates, we rely heavily on equations (11) and (2).

(ii) Observe that

$$
\begin{aligned}
K_{S} \cdot \sum_{\sigma \in G} D_{\sigma} & =K_{S} \cdot\left(\left(p^{2}+p+1+\sum_{\sigma \in F \backslash\left\{\overrightarrow{0}, \vec{e}_{1}\right\}}\left(q+m_{\sigma}\right)\right) H-(p+1) \sum_{i=1}^{p^{2}+p+1} E_{i}\right) \\
& =-3\left(p^{2}+p+1+\sum_{\sigma \in F \backslash\left\{\overrightarrow{0}_{\vec{e}}\right\}}\left(q+m_{\sigma}\right)\right)+(p+1)\left(p^{2}+p+1\right) \\
& =p^{3}+O\left(p^{2}\right) .
\end{aligned}
$$

(iii) Similarly, we calculate

$$
\begin{aligned}
\left(\sum_{\sigma \in G} D_{\sigma}\right)^{2} & =\left(p^{2}+p+1+\sum_{\sigma \in F \backslash\left\{\overrightarrow{0}, \vec{e}_{1}\right\}}\left(q+m_{\sigma}\right)\right)^{2}-(p+1)^{2}\left(p^{2}+p+1\right) \\
& =\left(p^{4}+2 p^{3}+O\left(p^{2}\right)\right)-\left(p^{4}+3 p^{3}+O\left(p^{2}\right)\right) \\
& =-p^{3}+O\left(p^{2}\right) .
\end{aligned}
$$


(iv) Lastly, by equation (2) we have

$$
\begin{aligned}
\sum_{\chi \in G^{\vee}} L_{\chi} \cdot( & \left.L_{\chi}+K_{S}\right)=\sum_{\gamma \in G} L_{\chi_{\gamma}} \cdot\left(L_{\chi_{\gamma}}+K_{S}\right) \\
= & \frac{1}{q^{2}} \sum_{\gamma \in G}\left[-3 q \gamma_{1}\left(p^{2}+p+1\right)+\gamma_{1}^{2}\left(p^{2}+p+1\right)^{2}\right. \\
& +2 \gamma_{1}\left(p^{2}+p+1\right) f(\gamma, \sigma)-3 q f(\gamma, \sigma)+f(\gamma, \sigma)^{2} \\
& \left.+\gamma_{1}(p+1)\left(q-\gamma_{1}(p+1)\right)\left(p^{2}+p+1\right)\right] \\
= & \frac{1}{q^{2}} \sum_{\gamma \in G}\left[\gamma_{1}^{2}\left(p^{2}+p+1\right)^{2}+\gamma_{1}(p+1)\left(q-\gamma_{1}(p+1)\right)\left(p^{2}+p+1\right)\right]+O\left(p^{2}\right) \\
= & \frac{1}{q^{2}}\left[\left(p^{4}+2 p^{3}\right) \sum_{\gamma \in G} \gamma_{1}^{2}+p^{3} q \sum_{\gamma \in G} \gamma_{1}-\left(p^{4}+3 p^{3}\right) \sum_{\gamma \in G} \gamma_{1}^{2}\right]+O\left(p^{2}\right) \\
= & \frac{1}{q^{2}}\left[-\sum_{\gamma \in G} \gamma_{1}^{2}+q \sum_{\gamma \in G} \gamma_{1}\right] p^{3}+O\left(p^{2}\right) \\
= & {\left[-\frac{1}{q^{2}} \sum_{0 \leq \gamma_{1}, \ldots, \gamma_{n} \leq q-1} \gamma_{1}^{2}+\frac{1}{q} \sum_{0 \leq \gamma_{1}, \ldots, \gamma_{n} \leq q-1} \gamma_{1}\right] p^{3}+O\left(p^{2}\right) } \\
= & {\left[-\frac{1}{q^{2}}\left(q^{n-1} \frac{q(q-1)(2 q-1)}{6}\right)+\frac{1}{q}\left(q^{n-1} \frac{q(q-1)}{2}\right)\right] p^{3}+O\left(p^{2}\right) } \\
= & {\left[\frac{1}{6} q^{n-2}\left(q^{2}-1\right)\right] p^{3}+O\left(p^{2}\right) . }
\end{aligned}
$$

We're now in a position to quickly prove the proposition.

Proof. Recall that

$$
K_{\tilde{S}}^{2}=q^{n}\left(K_{S}+\frac{q-1}{q} \sum_{\sigma} D_{\sigma}\right)^{2} .
$$

Applying parts (i)-(iii) of Lemma 3 then immediately yields

$$
K_{\tilde{S}}^{2}=q^{n-2}\left(q^{2}-1\right) p^{3}+O\left(p^{2}\right) .
$$

Similarly, we have

$$
\begin{aligned}
\chi\left(\mathcal{O}_{\tilde{S}}\right) & =q^{n} \chi\left(\mathcal{O}_{S}\right)+\frac{1}{2} \sum_{\chi} L_{\chi} \cdot\left(L_{\chi}+K_{S}\right) \\
& =\frac{1}{12} q^{n-2}\left(q^{2}-1\right) p^{3}+O\left(p^{2}\right) .
\end{aligned}
$$

The result follows.

The following is now immediate.

Corollary. For each prime $q \geq 3$ and all sufficiently large primes $p \equiv-1(\bmod q)$, there exists a surface $\tilde{S}$ in characteristic p, nonsingular and of general type, with $\frac{K_{\tilde{S}}^{2}}{\chi\left(\mathcal{O}_{\tilde{S}}\right)}>9$. 
Future considerations. R. Pardini has posed several interesting questions regarding the minimality of these surfaces and variations of the construction. The minimality of the surfaces remains to be seen. As for the construction, the requirement $p \equiv-1(\bmod q)$ seems quite arbitrary. It is likely that variations of this construction could produce similar surfaces for arbitrary primes $p$.

\section{ACKNOWLEDGEMENTS}

I thank R. Vakil for suggesting this strategy of constructing interesting surfaces in positive characteristic, as well as providing insight throughout the process. His guidance has been invaluable.

I also thank R. Pardini for her many helpful suggestions, and especially for discovering a serious error in the original draft of this paper.

\section{REFERENCES}

[B] F.A. Bogomolov, Holomorphic tensors and vector bundles on projective manifolds, Math. USSR-Izv. 13 (1979), 499-555. MR0522939 (80j:14014)

[C] F. Catanese, On the moduli spaces of surfaces of general type, J. Diff. Geom. 19 (1984), 483-515. MR755236 (86h:14031)

[H1] F. Hirzebruch, Automorphe Formen und der Satz von Riemann-Roch, in Symposium Internacional de Topologia Algebraica, Mexico (1958). MR0103280(21:2058)

[H2] - Arrangements of lines and algebraic surfaces, in Arithmetic and Geometry, Vol. II, Progr. Math. 36, Birkhäuser, Boston, Mass. (1983), 113-140. MR717609 (84m:14037)

[H3] _ Chern numbers of algebraic surfaces: an example, Math. Ann. 266, no. 3 (1984), 351-356. MR730175 (85j:14069)

[L] W.E. Lang, Examples of surfaces of general type with vector fields, in Arithmetic and Geometry, Vol. II, Progr. Math. 36, Birkhäuser, Boston, Mass. (1983), 167-173. MR717611 (86f:14022)

[M1] M. Manetti, On the moduli space of diffeomorphic algebraic surfaces, Invent. Math. 143 (2001), 29-76. MR.1802792 (2001j:14050)

[M2] Y. Miyaoka, On the Chern numbers of surfaces of general type, Invent. Math. 42 (1977), 225-237. MR0460343 (57:337)

[P] R. Pardini, Abelian covers of algebraic varieties, J. Reine Angew. Math. 417 (1991), 191-213. MR.1103912 (92g:14012)

[R] M. Reid, Bogomolov's theorem $c_{1}^{2} \leq 4 c_{2}$, in Proceedings of the International Symposium on Algebraic Geometry, Kinokuniya Book Store, Tokyo (1978), 623-642. MR578877|(82b:14014)

[V] R. Vakil, Murphy's law in algebraic geometry: Badly-behaved deformation spaces, Invent. Math., to appear.

[Y] S.-T. Yau, Calabi's conjecture and some new results in algebraic geometry, Proc. Natl. Acad. Sci. USA 74 (1977), 1798-1799. MR0451180 (56:9467)

Department of Mathematics, University of Utah, Salt Lake City, Utah 84102

E-mail address: easton@math.utah.edu 